\title{
Review of minimizing a vertical handover in a heterogeneous wireless network.
}

\begin{abstract}
Nowadays many different types of networks communicate among themselves to form heterogeneous networks. Vertical handovers between them are required to supply ongoing internet access to mobile nodes who switch from one coverage area to another with different characteristics. Mobility management techniques between heterogeneous network are necessary to reduce latency time and professionally treat the insufficient radio access resources to indemnity specific quality of service. This paper reviews literatures that are related to minimizing a vertical handover in heterogeneous wireless networks. This paper reviews literatures that are related to minimizing a vertical handover in heterogeneous wireless networks. This review investigated various handover management technologies for providing pure mobility between different access techniques such as GPRS, UMTS, and WIFI. More of these solutions used mobile IP (MIP), transmission control protocol (TCP), stream control transmission protocol (SCTP) and session initiation protocol (SIP) to support integration between WLAN and UMTS. From the review we conclude that SCTP is much more robust against packet loss and delay compared to TCP, SIP, and MIP. This fact makes SCTP a potential scheme for heterogeneous wireless networks.
\end{abstract}

Keyword: $3 \mathrm{G}$ network; Integrating wireless local area networks/universal mobile telecommunications system networks; Mobility management; Vertical handover. 